\title{
Insulintherapie bei der Katze - Tipps für die richtige Insulindosis
}

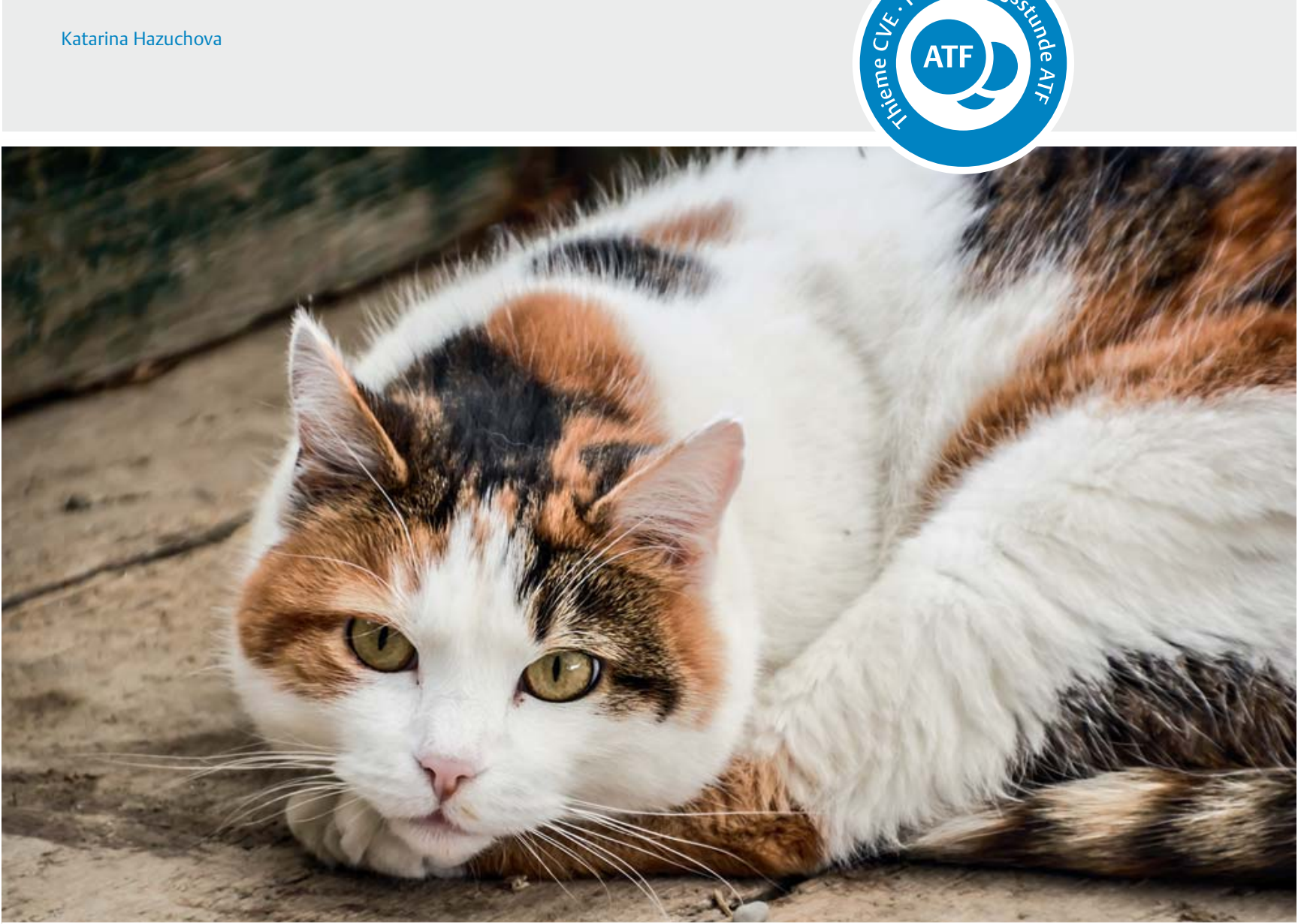

(c) badhog - stock.adobe.com

Das Management des Diabetes mellitus ist komplex und bedarf einer engen Zusammenarbeit zwischen Tierarzt und Tierbesitzer. Die Insulintherapie stellt die Grundlage der Diabetesbehandlung bei der Katze dar. Wenn möglich, sollte die Insulintherapie durch die Fütterung einer kohlenhydratarmen, proteinreichen Diät unterstützt werden.

\section{Insulin}

Seitdem Insulin zum ersten Mal im Jahr 1921 durch Banting und Best aus den Langerhans-Inseln eines Hundes extrahiert wurde, sind schon fast 100 Jahre vergangen. Verschiedene Insulinpräparate stehen heutzutage zur Diabetes-Behandlung des Menschen zur Verfügung, aber nur 2 Präparate sind für die Katze zugelassen ( $\bullet$ Abb. 1).

\section{Welche Insulinarten gibt es und wie unterscheiden sie sich?}

Insuline werden anhand ihrer Wirkdauer als kurz wirkende Normalinsuline, Intermediärinsuline, Langzeitinsuline (beim Menschen auch als „Basalinsuline“ bekannt) und sog. Mischinsuline, die aus einer Mischung (in verschiedenen Verhältnissen) von kurz und lang wirkendem Insulin bestehen, klassifiziert. Mischinsuline werden bei der Katze nicht verwendet und sind hier daher nicht weiter beschrieben. Informationen zu Normalinsulinen, die zur Be- 


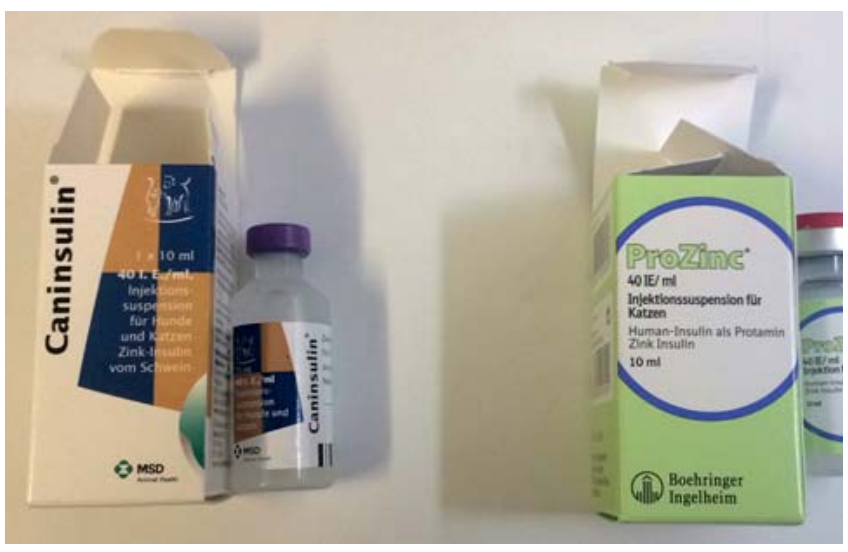

- Abb. 1 Caninsulin (MSD Tiergesundheit) und ProZinc (Boehringer Ingelheim), die 2 bei der Katze zugelassenen Insulinpräparate. (c) Katarina Hazuchova

handlung der diabetischen Ketoazidose gebraucht werden, sind anderen Literaturquellen zu entnehmen [1].

Die allgemeine Regel ist, dass Insulin-Präparate mit kürzerer Wirkdauer potenter sind, d. h. der Wirkungseintritt ist schneller und der Glukosespiegel wird tiefer gesenkt im Vergleich zu Intermediär- und Langzeitinsulinen. Die längere Wirkdauer von Intermediär- und Langzeitinsulinen wird in der Regel durch eine verlangsamte Absorption aus dem subkutanen Gewebe erreicht. Um dies zu gewährleisten, werden entweder inerte Stoffe wie Protamin und Zink zugefügt oder Modifikationen des Insulinmoleküls vorgenommen ( Tab. 1 ). Das Insulinanalogon Detemir wird zusätzlich an Albumin gebunden, das die Halbwertszeit dieses Insulins verlängert.

\section{Welche Insulinpräparate stehen zur Verfügung?}

\section{Caninsulin ${ }^{\circledR}$ und ProZinc ${ }^{\circledR}$}

Zwei Insulinpräparate sind für die Behandlung des Diabetes bei der Katze zugelassen - Caninsulin ${ }^{\circledR}$ (MSD Tiergesundheit) und ProZinc ${ }^{\circledR}$ (Boehringer Ingelheim):

- Caninsulin ${ }^{\circledR}$, ein porcines Lente-Insulin, weist bei der Katze eine intermediäre Wirkdauer auf.

- ProZinc ${ }^{\circledR}$, ein humanes rekombinantes Protamin-ZinkInsulin, ist ein Langzeitinsulin.

Obwohl die aktuellen Leitlinien zur Behandlung des Diabetes der Katze den Einsatz von Langzeitinsulinen als Mittel der 1 . Wahl empfehlen [2,3] und ProZinc ${ }^{\circledR}$ das einzige zugelassene Langzeitinsulin-Präparat ist, besteht rechtlich und medizinisch kein Grund, Katzen, die mit Caninsulin ${ }^{\circledR}$ gut eingestellt sind, auf ProZinc ${ }^{\circledR}$ umzustellen.

\section{Glargin und Detemir}

Vor der Einführung von ProZinc ${ }^{\circledR}$ wurden von vielen Tierärzten die nicht zugelassenen humanmedizinischen Insulinanaloga Glargin und Detemir eingesetzt. Die Rechtfertigung für deren Umwidmung und den Einsatz als Erstlinientherapie in der Vergangenheit war, dass keine zugelassenen tiermedizinischen Langzeitinsuline verfügbar waren. Zudem haben die oben angegebenen Leitlinien [2,3] für die Behandlung des Katzendiabetes eindeutig Langzeitinsuline gegenüber dem zugelassenen intermediären Lente-Insulin (Caninsulin ${ }^{\circledR}$ ) vorgezogen. Die Empfehlung der Leitlinien war durch Expertenmeinung und einige Studien belegt [4-6]. Da aber momentan ein für die Katze zugelassenes Langzeitinsulin vorhanden ist und eine aktuelle klinische Studie gezeigt hat, dass es keinen Unterschied zwischen Insulin Glargin und ProZinc ${ }^{\circledR}$ in Bezug auf die glykämischen Parameter oder Remissions-

- Tab. 1 Eigenschaften der bei der Katze verwendeten Insulinpräparate.

\begin{tabular}{|c|c|c|c|c|}
\hline $\begin{array}{l}\text { Markenname } \\
\text { (Hersteller) }\end{array}$ & Wirkstoff & $\begin{array}{l}\text { Konzentra- } \\
\text { tion IE/ml }\end{array}$ & Eigenschaften & $\begin{array}{l}\text { Aufbewahrung (Hersteller- } \\
\text { angaben) }\end{array}$ \\
\hline $\begin{array}{l}\text { ProZinc }{ }^{\circledR} \text { (Boehringer } \\
\text { Ingelheim) }\end{array}$ & $\begin{array}{l}\text { humanes rekom- } \\
\text { binantes Prota- } \\
\text { min-Zink-Insulin }\end{array}$ & 40 & $\begin{array}{l}\text { Protamin-Zink verlangsamt Resorption aus } \\
\text { der Unterhaut }\end{array}$ & $\begin{array}{l}\text { Lagern bei } 2-8^{\circ} \mathrm{C} \text {; nach } \mathrm{An}- \\
\text { bruch } 60 \text { Tage haltbar }\end{array}$ \\
\hline $\begin{array}{l}\text { Caninsulin }{ }^{\circledR} \text { (MSD } \\
\text { Tiergesundheit) }\end{array}$ & $\begin{array}{l}\text { porcines Lente- } \\
\text { Insulin }\end{array}$ & 40 & $\begin{array}{l}\text { 30-35\% amorphes kurzwirkendes Insulin } \\
+65-70 \% \text { kristallines Langzeitinsulin }\end{array}$ & $\begin{array}{l}\text { Lagern bei } 2-8^{\circ} \mathrm{C} \text {; nach } \mathrm{An} \text { - } \\
\text { bruch } 42 \text { Tage haltbar }\end{array}$ \\
\hline Lantus $^{\circledR}$ (Sanofi) & Glargin & 100 & $\begin{array}{l}\text { Aminosäure Asparagin ist auf der Position } \\
21 \text { in der Kette A durch Glycin ersetzt wor- } \\
\text { den und } 2 \text { Aminosäuren wurden am C-Ter- } \\
\text { minus der Kette B angehängt, was den } \\
\text { isoelektrischen Punkt verschiebt und die } \\
\text { Löslichkeit in der Unterhaut vermindert }\end{array}$ & $\begin{array}{l}\text { nicht angebrochen bei } 2-8^{\circ} \mathrm{C} \text {, } \\
\text { nach Anbruch bei Raumtem- } \\
\text { peratur }\left(<30^{\circ} \mathrm{C}\right) \text { lagern; nach } \\
\text { Anbruch } 30 \text { Tage haltbar }\end{array}$ \\
\hline $\begin{array}{l}\text { Levemir }^{\circledR} \text { (Novo } \\
\text { Nordisk) }\end{array}$ & Detemir & 100 & $\begin{array}{l}\text { Aminosäure Threonin ist entfernt worden } \\
\text { von der Position } 30 \text { der B-Kette und Myris- } \\
\text { tyl-Säure ist angehängt worden an der } \\
\text { Position } 29 \text { der B-Kette, was eine Bindung } \\
\text { an Albumin ermöglicht }\end{array}$ & $\begin{array}{l}\text { nicht angebrochen bei } 2-8^{\circ} \mathrm{C} \text {, } \\
\text { nach Anbruch bei Raumtem- } \\
\text { peratur oder im Kühlschrank } \\
\text { lagern; nach Anbruch } 42 \text { Tage } \\
\text { haltbar }\end{array}$ \\
\hline
\end{tabular}


raten gibt [7], besteht auch medizinisch kein Grund mehr, Insulin Glargin als Erstlinientherapie anzuwenden.

\section{Einige pharmakologische Fakten}

Die Eigenschaften der Insulinpräparate, inklusive der nicht zugelassenen Insulinanaloga, sind in der $>$ Tab. 1 aufgelistet. Auf die pharmakologischen Daten zur Wirkdauer wird in diesem Artikel verzichtet, da diese lediglich bei einer kleinen Anzahl gesunder Katzen und nur selten bei diabetischen Katzen untersucht worden sind. Zudem wurden in den Studien verschiedene Methoden angewandt, was einen Vergleich der Daten erschwert [8-11]. Obwohl in manchen Studien Glargin und Protamin-ZinkInsulin eine Wirkdauer von fast 24 Stunden hatten [9, 10] und daher potenziell 1-mal täglich eingesetzt werden könnten, ist dies bei den klinischen Patienten nur sehr selten der Fall. Die Studien mit der Goldstandard-Methode (euglykämische Clamp) konnten weder für Glargin noch für Detemir diese lange Wirkdauer bestätigen [11].

Im Praxisalltag sind die Angaben aus den pharmakologischen Studien leider nur wenig hilfreich, da das Ansprechen auf die Insulintherapie, Insulin-Wirkungseintritt, Nadir sowie die Wirkdauer von Katze zu Katze unterschiedlich sind und auch für das individuelle Tier von Tag zu Tag variieren können [12].

\section{Insulineinstellung}

\section{Welche Startdosis soll ich nehmen?}

In der Literatur wird meistens eine Insulin-Startdosis von 0,25-0,5 IE/kg q $12 \mathrm{~h}$ empfohlen. Man sollte eher an der unteren Grenze des Dosisspektrums anfangen, um Hypoglykämien zu vermeiden.

Merke

Eine „gute“ Anfangsdosis liegt somit bei der Katze bei $1 \mathrm{IE} /$ Katze q $12 \mathrm{~h}$.

Auch bei sehr großen Katzen sollte die Dosis 2 IE/Katze q $12 \mathrm{~h}$ nicht überschreiten. Manche Tierärzte bevorzugen, die Katze am 1. Behandlungstag in der Praxis zu überwachen. Mit diesem Vorgehen soll keine optimale Insulineinstellung erzielt werden, sondern es sollen Hypoglykämien erkannt werden. Die Insulindosis sollte an diesem 1. Behandlungstag auch bei einem ausbleibenden Effekt auf keinen Fall erhöht werden.

\section{Was muss der Besitzer wissen?}

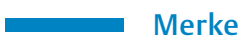

Bei der Entlassung in die häusliche Obhut ist eine ausführliche Besitzeraufklärung äußerst wichtig.

Die Besitzer sollten das Aufziehen von Insulin (zu Übungszwecken eignet sich Kochsalzlösung) und die Injektions- technik zusammen mit dem Tierarzt oder der TFA üben. Es ist hilfreich, den Besitzer auf nützliche Internet-Videos zu verweisen (s. Kasten am Artikelende).

Man sollte sicherstellen, dass der Besitzer weiß, ...

- wie Insulin aufzubewahren ist (aufrechtstehend im Kühlschrank, nicht in der Kühlschranktür),

- wie lange das Insulin-Fläschchen nach dem Anbruch haltbar ist (für Hersteller-Angaben siehe $>$ Tab. 1),

- wie man das Insulin vor der Verabreichung aufbereiten sollte (Caninsulin ${ }^{\circledR}$ „schütteln“, ProZinc ${ }^{\circledR}$ „,rollen“),

- welche Spritzen (40 IE-Spritzen mit beiden für die Katze zugelassenen Insulinen) zu verwenden sind und

- wie die Spritzen zu entsorgen sind.

Oft wird man von den Besitzern gefragt, ob sie die Insulin-Fläschchen auch länger nach dem Anbruch als vom Hersteller angegeben verwenden dürfen. Obwohl der Autorin dieses Artikels keine Stabilitätsstudien bekannt sind, wird in den aktuellen Leitlinien angegeben, dass die angebrochenen Fläschchen bis zu 3-6 Monate nach dem Anbruch verwendet werden können, solange keine makroskopischen Veränderungen (z.B. Farbveränderungen, „Flöckchen“) zu beobachten sind und eine adäquate glykämische Kontrolle gewährleistet wird [3].

\section{Wann erfolgt die erste Kontrolluntersuchung?}

Merke

Die erste Kontrolluntersuchung wird 5-7 Tage nach dem Therapiebeginn empfohlen.

Bei dieser sowie jeder weiteren Kontrolluntersuchung sollte das Ansprechen auf die Insulintherapie an erster Stelle anhand einer Beurteilung der klinischen Symptomatik erfolgen. Hierzu sind sog. Scoring-Systeme nützlich, die eine Standardisierung ermöglichen. Dies ist besonders hilfreich, wenn mehrere Tierärzte an der Behandlung beteiligt sind. Ein Beispiel für ein kürzlich veröffentlichtes Scoring-System zeigt $>$ Tab. 2.

Wenn möglich, sollte bei der ersten Kontrolluntersuchung in der Praxis auch ein Blutzuckertagesprofil (BZTP) angefertigt werden, wobei diese Technik früher oder später von ungefähr 75\% der Besitzer erlernt werden kann [13]. Heutzutage kann das Anfertigen des Blutzuckertagesprofils durch die Anwendung der sog. kontinuierlichen Glukosemonitoring-Systeme erleichtert werden. Obwohl Geräte wie das FreeStyle Libre von Abbott für die Katze noch nicht validiert sind, werden diese von vielen Tierärzten verwendet und die Erfahrungsberichte sind durchaus positiv.

Sollte die Anfertigung eines Blutzuckertagesprofils nicht möglich sein (z.B. sehr unkooperative Katzen, Kostengründe), kann alternativ eine Fruktosamin-Messung durchgeführt werden. Der Nachteil ist, dass diese keine 
- Tab. 2 Scoring-System zur Beurteilung der klinischen Symptomatik diabetischer Katzen. Jedem der 4 Leitsymptome des Diabetes wird anhand des Schweregrads der Symptome eine Punktzahl von 0 (nicht vorhanden) bis 3 (hochgradig) erteilt. Eine Gesamtpunktzahl von 0-12 ergibt den Schweregrad der Symptomatik (niedrige Gesamtzahl - weniger Symptome und umgekehrt) Gostelow et al. 2017 [15].

\begin{tabular}{|c|c|c|}
\hline klinisches Symptom & Schweregrad & Punktzahl \\
\hline $\begin{array}{l}\text { unbeabsichtigter Gewichtsverlust } \\
\text { über die letzten } 2 \text { Monate }\end{array}$ & $\begin{array}{l}\text { - nicht vorhanden oder Gewichtszunahme } \\
\text { - geringgradig (<5\% Verlust) } \\
\text { - mittelgradig ( } 5-10 \% \text { Verlust) } \\
\text { - hochgradig (> } 10 \% \text { Verlust) }\end{array}$ & 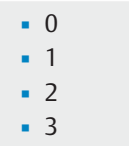 \\
\hline $\begin{array}{l}\text { erhöhte Wasseraufnahme und/oder } \\
\text { erhöhter Harnabsatz }\end{array}$ & $\begin{array}{l}\text { - normal } \\
\text { " geringgradig - etwas erhöht } \\
\text { " mittelgradig - der Wassernapf muss häufiger gefüllt werden } \\
\text { - hochgradig - trinkt die ganze Zeit }\end{array}$ & $\begin{array}{l}.0 \\
.1 \\
.2 \\
.3\end{array}$ \\
\hline gesteigerter Appetit & $\begin{array}{l}\text { - normal oder vermindert* } \\
\text { - geringgradig - frisst alles gierig auf } \\
\text { - mittelgradig - frisst alles gierig auf und bettelt für mehr } \\
\text { - hochgradig - ist besessen von Futter }\end{array}$ & $\begin{array}{l}.0 \\
.1 \\
.2 \\
.3\end{array}$ \\
\hline verminderte Aktivität & $\begin{array}{l}\text { " normal oder erhöht } \\
\text { - geringgradig vermindert - etwas weniger aktiv } \\
\text { - mittelgradig vermindert - deutlich weniger aktiv } \\
\text { - hochgradig vermindert - liegt die meiste Zeit }\end{array}$ & $\begin{array}{l}.0 \\
.1 \\
.2 \\
.3\end{array}$ \\
\hline Gesamtpunktzahl & - & $/ 12$ \\
\hline
\end{tabular}

Aussage bezüglich der Insulin-Wirkdauer oder des Nadirs erlaubt.

Es ist nicht zu empfehlen, die Insulindosis anhand von Einzelglukosemessungen zum Zeitpunkt des geschätzten Nadirs oder von Uringlukosebestimmungen anzupassen. Wie bereits erwähnt, variiert der Nadir-Zeitpunkt sogar bei demselben Tier [12]. Wird nur eine einzige Glukosemessung durchgeführt, besteht die Gefahr, diesen Zeitpunkt zu verpassen. Einzelglukosemessungen vor der Insulingabe können bei Katzen mit seit lange bestehendem Diabetes nützlich sein, solange die Katze keine Symptome des Diabetes aufweist und die Glukose vor der Insulingabe bei einem Wert zwischen 10 und $15 \mathrm{mmol} / \mathrm{l}$ liegt. Sollte das jedoch nicht der Fall sein und/oder sollte die Katze Symptome des Diabetes entwickelt haben, ist die Durchführung eines Blutzuckertagesprofils notwendig. Die Insulindosis anhand der Glukosurie anzupassen ist auch schwierig, da Glukose im Urin den Blutglukose-Spiegel über mehrere Stunden widerspiegelt und das Vorliegen einer Glukosurie hypoglykämische Phasen nicht ausschließt.

\section{Wie wird die Insulindosis angepasst?}

Merke

Im Idealfall wird die Insulindosis anhand der Kombination von klinischer Symptomatik ( $\vee$ Tab. 2 ) und der Resultate eines Blutzuckertagesprofils angepasst.

Das Ziel der Insulin-Behandlung ist, den Glukosespiegel unter $15 \mathrm{mmol} / \mathrm{I}$ (= Nierenschwelle für Glukose) zu hal- ten, was zur Linderung und Behebung der klinischen Symptome führen sollte. Entscheidend und limitierend für die Dosisanpassung ist der Glukose-Nadir, der zwischen 4,5 und $8 \mathrm{mmol} / \mathrm{I}$ liegen sollte $[2,3,14]$. In Ausnahmefällen und bei gut eingestellten Patienten sind Glukosewerte von 3,5-4,5 mmol/l zu tolerieren, aber Glukosewerte $<3,5 \mathrm{mmol} / \mathrm{l}$ sollten in jedem Fall zu einer Insulindosisreduktion führen.

\section{Merke}

Die Insulindosis sollte schrittweise angepasst werden.

Die Dosiserhöhung findet in der Regel in Schritten von 0,5 IE/Injektion (selten $1 \mathrm{IE} /$ Injektion) alle 5-7 Tage statt (je nach Monitoring-Möglichkeiten des Besitzers kann das Intervall länger sein, aber nicht kürzer). Eine Dosisreduktion kann deutlich schneller erfolgen und geschieht immer dann, wenn eine biochemische (asymptomatische) oder klinische Hypoglykämie festgestellt wird. In der Regel ist eine Dosisreduktion um 0,5-1 IE/Injektion ausreichend [15], obwohl man früher bei Hypoglykämien eine $50 \%$ ige Dosisreduktion empfohlen hat.

\section{Wie lange dauert die Stabilisierung eines Diabetikers?}

In der Regel dauert es einige Wochen bis zu 2-3 Monate, um einen Diabetiker zu stabilisieren. Der Besitzer sollte über diesen Zeitrahmen aufgeklärt werden, um Frustration zu vermeiden. Die meisten diabetischen Katzen bedürfen langfristig einer Insulindosis von 0,5-3 IE/Injektion/Katze q 12 h [14]. In der Stabilisierungsphase ist es 


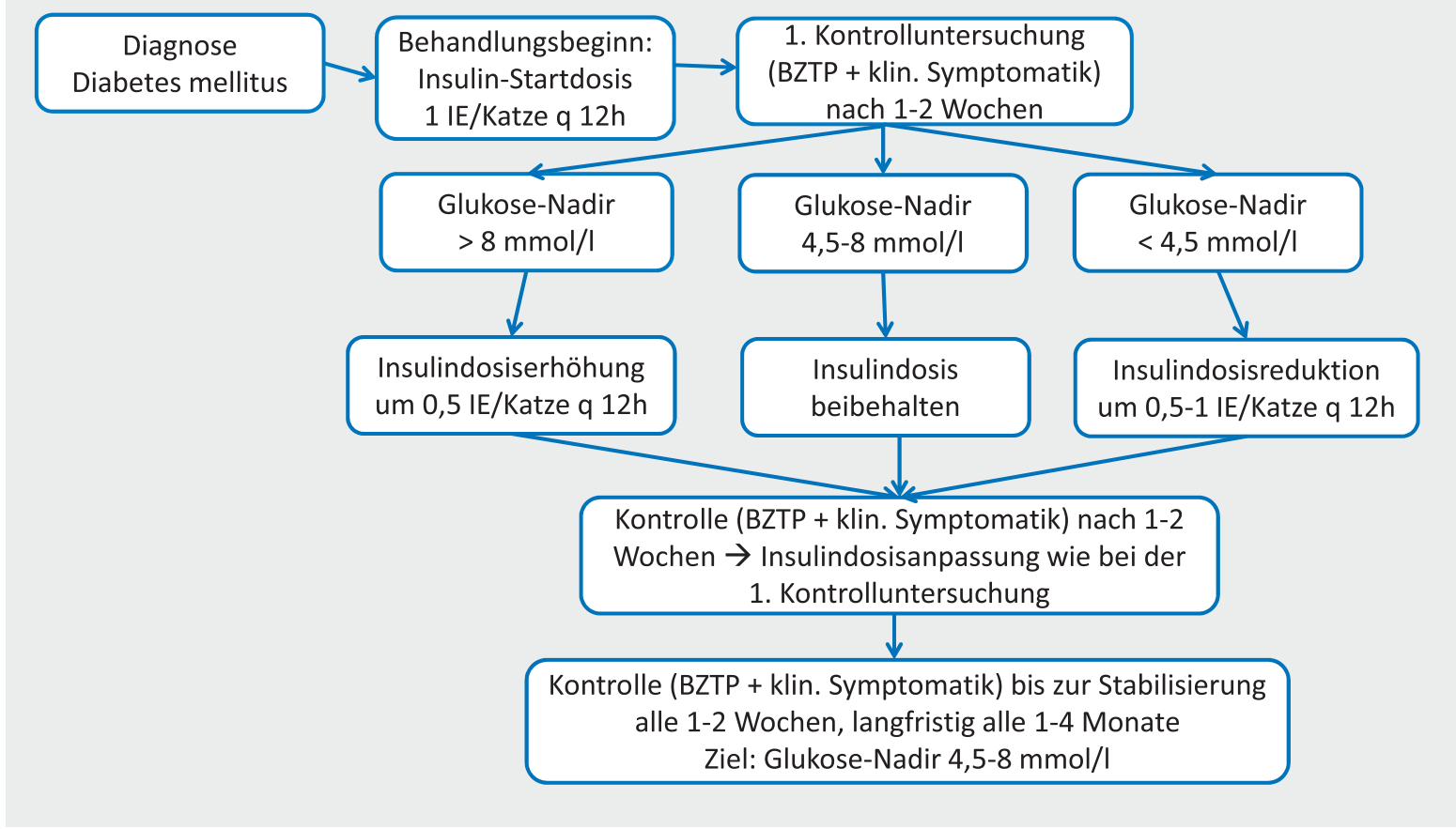

- Abb. 2 Ein Schema zur Insulindosis-Anpassung anhand von Ergebnissen der Blutzuckertagesprofile (BZTP). @ Katarina Hazuchova

aber nicht ungewöhnlich, dass man auch 4-5 IE/Injektion/Katze q $12 \mathrm{~h}$ benötigt. Katzen mit Begleiterkrankungen (z. B. Adipositas, entzündliche Erkrankungen) können auch langfristig höhere Dosen benötigen. Bei einigen Katzen kann es notwendig sein, nach der initialen Dosissteigerung die Dosis zu reduzieren, möglicherweise aufgrund des Rückgangs der Glukosetoxizität. Gestörte Insulinsekretion und Apoptose der $\beta$-Zellen als Folge einer Hyperglykämie wurden beim Mensch und in einer experimentellen Studie auch bei der Katze gezeigt [16]. Rückgang der Glukosetoxizität ist eventuell auch einer der Faktoren, die die diabetische Remission begünstigen [17]. Da die benötigte Insulindosis mit der Zeit fluktuieren kann, ist auch nach der Stabilisierungsphase eine Therapieüberwachung notwendig, in der Regel kann aber die Frequenz auf alle 1-4 Monate verlängert werden. Ein Schema zum Monitoring und zur Insulindosisanpassung bietet $\mathbf{A b b} \mathbf{2}$.

\section{Was ist bei einem Wechsel des Insulins zu beachten?}

Sollte im Laufe der Behandlung eine Umstellung von Caninsulin ${ }^{\circledR}$ auf ProZinc ${ }^{\circledR}$ aus medizinischer Sicht indiziert sein (z.B. aufgrund einer zu kurzen Wirkdauer bei Caninsulin $^{\circledR}$-behandelten Katzen), so hat sich die vom Hersteller angegebene Anfangsdosis von 0,2-0,7 IE $/ \mathrm{kg}$ (im Median 0,5 IE $/ \mathrm{kg}$ ) q $12 \mathrm{~h}$ für die Umstellung in einer klinischen Studie als geeignet erwiesen [15].
Studien zur Umstellung von ProZinc ${ }^{\circledR}$ auf Caninsulin ${ }^{\circledR}$ liegen zurzeit nicht vor, aber in einer kürzlich publizierten Studie wurde von 2 Katzen berichtet, die mit Caninsulin ${ }^{\circledR}$ eine Insulin-Wirkdauer von $>9 \mathrm{~h}$ hatten, die nach der Umstellung auf ProZinc ${ }^{\circledR}$ auf $<9$ h abfiel [15]. Daher kann bei einzelnen Katzen eine Umstellung von ProZinc ${ }^{\circledR}$ auf Caninsulin ${ }^{\circledR}$ indiziert sein, wenn mit ProZinc ${ }^{\circledR}$ keine ausreichende glykämische Kontrolle erreicht werden kann.

\section{Welche Nebenwirkungen sind zu erwarten?}

\section{Hypoglykämie}

Die häufigste Nebenwirkung der Insulintherapie ist eine Hypoglykämie. Obwohl immer wieder diskutiert wird, dass die Behandlung mit den Insulinanaloga Glargin und Detemir mit einer geringeren Anzahl an symptomatischen Hypoglykämien vergesellschaftet ist und trotz häufig aufgetretenen asymptomatischen Hypoglykämien sicher ist [4,5], gibt es nur wenige Vergleichsstudien mit anderen Insulinen.

In einer randomisierten klinischen Studie zeigte sich kein Unterschied in Bezug auf asymptomatische und klinische Hypoglykämien zwischen Katzen, die mit Glargin und ProZinc ${ }^{\circledR}$ behandelt wurden [18]. In einem kürzlich herausgegebenen Bericht wurde von 30 klinischen hypoglykämischen Episoden bei 28 diabetischen Katzen berichtet, von denen 13 mit Glargin behandelt wurden [19]. 
Die häufigsten Ursachen der Hypoglykämie waren eine verminderte Futteraufnahme oder Erbrechen und eine versehentliche Insulin-Überdosierung. Bei der Hälfte der hypoglykämischen Episoden blieb die Ursache unbekannt. Fast alle Katzen hatten neurologische Symptome von verschiedener Ausprägung und die meisten zeigten auch eine vorübergehende Erblindung. Mit entsprechender Behandlung (Glukose-Boli und Infusion) wurde die Hypoglykämie bei 18 Katzen innerhalb von $2 \mathrm{~h}$ behoben, in den restlichen Fällen hat es bis zu 4 Tagen gedauert. Katzen, deren Allgemeinbefinden und Neurostatus sich innerhalb von $12 \mathrm{~h}$ gebessert haben, wurden entlassen, während 7 Katzen, bei denen dies nicht der Fall war, euthanasiert wurden [19].

Zu den ersten Maßnahmen, die bei Katzen mit asymptomatischer Hypoglykämie oder bei Katzen mit minimalen Symptomen vom Besitzer eingeleitet werden sollten, gehört die Fütterung einer kohlenhydratreichen Diät, ggf. begleitet durch Verabreichung von Honig oder Glukoselösung. Bei schwerwiegenden Symptomen und wenn eine tierärztliche Behandlung notwendig ist, wird Glukose intravenös in Form von Boli (z. B. 40\%ige Glukose 1:1 verdünnt und $1-4 \mathrm{ml} / \mathrm{kg}$ über 5-10 Minuten) gefolgt von einer Glukoseinfusion (2,5-5\%ige Glukose) verabreicht.

\section{Somogyi-Effekt}

Viele Jahre ist man von einem sog. Somogyi-Effekt ausgegangen, der zu einer ausgeprägten und anhaltenden Hyperglykämie als Folge einer Gegenregulation der Stresshormone bei einer Hypoglykämie führt. Aktuelle Studien zeigen, dass dieses Phänomen wahrscheinlich sehr selten ist. Der typische Somogyi-Effekt (oder auch „rebound hyperglycemia“ = „gegenregulatorische Hyperglykämie“) wurde retrospektiv nur bei 4 von über 10000 Blutzuckertagesprofilen identifiziert [20]. In der Humanmedizin wurde stattdessen der Begriff „glykämische Variabilität“ eingeführt. Vor Kurzem wurde vorgeschlagen, bei der Katze die Bezeichnung „Somogyi-Effekt“ durch „posthypoglykämische Hyperglykämie“ zu ersetzen [21]. In einer kürzlich publizierten Studie wurde gezeigt, dass dieses Phänomen mit einer schlechteren glykämischen Kontrolle und niedrigeren Remissionsraten assoziiert war [21].

Andere Nebenwirkungen der Insulintherapie wie subkutane Reaktion an der Injektionsstelle [15] oder Antikörperbildung gegen das verwendete Insulin [22] sind selten und vermutlich klinisch nicht relevant.

\section{Warum schlägt die Insulintherapie bei meinem Patienten nicht an?}

Je nach Literaturquelle spricht man von einer Insulinresistenz, wenn Insulindosen > 1-1,5 IE/kg q 12 h notwendig sind, um den Diabetes zu kontrollieren.
An erster Stelle ist hier immer das Besitzer-Management zu überprüfen:

- Wird das Insulin zuverlässig verabreicht?

- Wo wird es gelagert?

- Könnte das Insulin abgelaufen sein?

Als nächster Schritt sollte das tierärztliche Management beurteilt werden. Wenn z. B. Einzelmessungen der Glukose zum Zeitpunkt des geschätzten Nadirs oder die Glukosurie als einzige Parameter zur Insulindosis-Anpassung herangezogen werden, können die Resultate dieser Tests fälschlicherweise den Eindruck erwecken, dass der Patient insulinresistent ist. Dabei könnte der Patient unter Umständen sogar hypoglykämische Phasen entwickelt haben. Nicht selten ist einfach auch die Insulindosis noch nicht ausreichend, da manche Patienten mehr Insulin brauchen als andere. Eine andere häufige Ursache ist eine zu kurze Dauer der Insulinbehandlung - wie bereits erwähnt, kann die Stabilisierungsphase einige Wochen bis ungefähr 3 Monate dauern.

Erst an 3. Stelle kommen als Ursache für einen schlecht einstellbaren Diabetes Erkrankungen, die mit einer Insulinresistenz oder wechselnder Insulinwirkung einhergehen. Dazu gehören:

- Adipositas

- andere Endokrinopathien (z. B. Akromegalie, Hyperthyreose, Hyperadrenokortizismus)

- entzündliche Erkrankungen (z. B. Pankreatitis, Gingivitis)

- chronische Nierenerkrankung

- Verabreichung von Medikamenten (Steroide oder Megestrolacetat)

Diese Erkrankungen sollten entsprechend abgeklärt und behandelt werden, ggf. sollten die hier genannten Medikamente abgesetzt oder ersetzt werden, wo möglich.

\section{Welches Insulin hat die höchsten Remissionsraten?}

Obwohl von hohen Remissionsraten in Studien mit den Insulinen Glargin oder Detemir berichtet wurde [4-6], haben diese Studien verschiedene Schwachstellen. Die einzige randomisierte klinische Studie, die Glargin und ProZinc ${ }^{\circledR}$ verglichen hat und bei der Katzen über 1 Jahr verfolgt wurde, konnte keinen Unterschied zwischen diesen 2 Insulinen in Bezug auf glykämische Parameter oder Remissionsraten identifizieren [7]. Randomisierte klinische Studien mit Caninsulin ${ }^{\circledR}$ und ProZinc ${ }^{\circledR}$ wurden bis jetzt nicht durchgeführt, aber zurzeit gibt es keine unvoreingenommene Studie, die eine Evidenz zugunsten eines spezifischen Insulinpräparats zeigen würde [17]. 


\section{Gibt es Alternativen, wenn eine Insulintherapie nicht möglich ist?}

Obwohl sich in der Humanmedizin die Inkretin-Behandlung (GLP-1-Analoga und DPP4-Inhibitoren) als sehr vielversprechend erwiesen hat, zeigte die bis jetzt einzige publizierte Studie, die Exenatid (GLP-1-Analogon) bei diabetischen Katzen in Kombination mit Insulin Glargin eingesetzt hat, keinen Vorteil im Vergleich zu der Kombination von Insulin Glargin und einem Placebo [23]. Die Inkretin-Therapie ohne gleichzeitige Insulinbehandlung wurde bei der Katze nicht getestet. Auch die oralen Antidiabetika Glipizide und Metformin scheinen bei der Katze wenig effektiv zu sein [24, 25].

Wenn möglich, sollte die Insulintherapie durch die Fütterung einer kohlenhydratarmen, proteinreichen Diät unterstützt werden. Ein Verzicht auf die Insulingaben ist durch eine alleinige Diätfütterung bislang nicht möglich.

Daher lässt sich leider nur sagen, dass derzeit keine Alternative zur Insulintherapie bei der Katze besteht.

TIPPS

\section{Webseiten}

- www.icatcare.org/advice/cat-health/diabetesmellitus (> 20 Sprachen zur Auswahl)

- www.prozinc.de (auf Deutsch)

- www.prozinc.co.uk (auf Englisch, ausführlichere Informationen)

- www.caninsulin.de

Die Diabetic Remission Clinic (RVC) bei Facebook: www.facebook.com/RVC.Diabetic.Remission.Clinic

\section{Videos}

- iCatCare - How to give your cat an insulin injection: www.youtube.com/watch?v=by4U2VFxOGg

- www.prozinc.co.uk (Resources > Managing your Cat's Diabetes > How to use ProZinc - Injecting your cat)

- www.caninsulin.de - Startseite: Gebrauch des VetPen ${ }^{\circledR}$

\section{App}

RVC Diabetes der Haustiere App (mit Diabetes-Tagebuch, Medikamenteninformationen, Wecker für Insulininjektionen, Versand des Tagesprofils an den Haustierarzt möglich; in mehreren Sprachen verfügbar)
Katarina Hazuchova, Dipl. ECVIM-CA MRCVS

The Royal Veterinary College

Hawkshead Lane

Hatfield

Hertfordshire

AL9 7TA

Großbritannien

khazuchova@rvc.ac.uk

\section{Literatur}

[1] Rudloff E. Diabetic ketoacidosis in the cat: Recognition and essential treatment. J Feline Med Surg 2017; 19 (11): 1167-1174

[2] Sparkes AH, Cannon M, Church D et al. ISFM consensus guidelines on the practical management of diabetes mellitus in cats. J Feline Med Surg 2015; 17 (3): 235-250

[3] Behrend E, Holford A, Lathan P et al. 2018 AAHA diabetes management guidelines for dogs and cats. J Am Anim Hosp Assoc 2018; 54 (1): 1-21

[4] Roomp K, Rand J. Intensive blood glucose control is safe and effective in diabetic cats using home monitoring and treatment with glargine. J Feline Med Surg 2009; 11 (8): 668-682

[5] Roomp K, Rand J. Evaluation of detemir in diabetic cats managed with a protocol for intensive blood glucose control. J Feline Med Surg 2012; 14 (8): 566-572

[6] Marshall RD, Rand JS, Morton JM. Treatment of newly diagnosed diabetic cats with glargine insulin improves glycaemic control and results in higher probability of remission than protamine zinc and lente insulins. J Feline Med Surg 2009; 11 (8): 683-691

[7] Gostelow R, Scudder C, Hazuchova K et al. One-year prospective randomized trial comparing efficacy of glargine and protamine zinc insulin in diabetic cats. 2017 ACVIM Forum Research Abstract Program. J Vet Intern Med 2017; 31 (4): 1273

[8] Martin G], Rand JS. Pharmacology of a $40 \mathrm{IU} / \mathrm{ml}$ porcine lente insulin preparation in diabetic cats: findings during the first week and after 5 or 9 weeks of therapy. J Feline Med Surg 2001; 3 (1): 23-30

[9] Marshall RD, Rand JS, Morton JM. Glargine and protamine zinc insulin have a longer duration of action and result in lower mean daily glucose concentrations than lente insulin in healthy cats. J Vet Pharmacol Ther 2018; 31 (3): 205-212

[10] Marshall RD, Rand JS, Morton JM. Insulin glargine has a long duration of effect following administration either once daily or twice daily in divided doses in healthy cats. J Feline Med Surg 2008; 10 (5): 488-494

[11] Gilor C, Ridge TK, Attermeier KJ et al. Pharmacodynamics of insulin detemir and insulin glargine assessed by an isoglycemic clamp method in healthy cats. J Vet Intern Med 2010; 24: 870 874

[12] Alt N, Kley S, Haessig M et al. Day-to-day variability of blood glucose concentration curves generated at home in cats with diabetes mellitus. J Am Vet Med Assoc 2007; 230 (7): 10111017

[13] Hazuchova K, Gostelow R, Scudder C et al. Acceptance of home blood glucose monitoring by owners of recently diagnosed diabetic cats and impact on quality of life changes in cat and owner. J Feline Med Surg 2018; 20 (8): 711-720

[14] Reusch C. Feline diabetes mellitus. In: Feldman EC, Nelson RW, Reusch C, Scott-Moncroie EB, eds. Canine and Feline Endocrinology. $4^{\text {th }}$ ed. St. Louis, Missouri: Elsevier; 2014: 258-314

[15] Gostelow R, Hazuchova K, Scudder C et al. Prospective evaluation of a protocol for transitioning porcine lente insulin-treat- 
ed diabetic cats to human recombinant protamine zinc insulin. J Feline Med Surg 2017; 20 (2): 114-121

[16] Zini E, Osto M, Franchini M et al. Hyperglycaemia but not hyperlipidaemia causes beta cell dysfunction and beta cell loss in the domestic cat. Diabetologia 2009; 52: 336-346

[17] Gostelow R, Forcada Y, Graves T et al. Systematic review of feline diabetic remission: separating fact from opinion. Vet J 2014; 202 (2): 208-221

[18] Gostelow R. Uncovering the recipe for diabetic remission in the cat [PhD thesis]. Royal Veterinary College; 2016

[19] Viebrock KA, Dennis ]. Hypoglycemic episodes in cats with diabetes mellitus: 30 cases (2013-2015). J Feline Med Surg 2018; 20 (6): 563-570

[20] Roomp K, Rand J. Rebound hyperglycaemia in diabetic cats. J Feline Med Surg 2016; 18 (8): 587-596

[21] Zini E, Salesov E, Dupont P et al. Glucose concentrations after insulin-induced hypoglycemia and glycemic variability in healthy and diabetic cats. J Vet Intern Med 2018; 32 (3): 978985

[22] Hoenig M, Reusch C, Peterson ME. Beta cell and insulin antibodies in treated and untreated diabetic cats. Vet Immunol Immunopathol 2000; 77 (1-2): 93-102

[23] Riederer A, Zini E, Salesov E et al. Effect of the glucagon-like peptide-1 analogue exenatide extended release in cats with newly diagnosed diabetes mellitus. J Vet Intern Med 2016; 30 (1): 92-100

[24] Nelson RW, Feldman EC, Ford SL et al. Effect of an orally administered sulfonylurea, glipizide, for treatment of diabetes mellitus in cats. J Am Vet Med Assoc 1993; 203 (6): 821-827

[25] Nelson R, Spann D, Elliott D et al. Evaluation of the oral antihyperglycemic drug metformin in normal and diabetic cats. J Vet Intern Med 2004; 18 (1): 18-24

Bibliografie

DOI https://doi.org/10.1055/a-0668-0728

Kleintier konkret 2018; 21: 19-28 @ Georg Thieme Verlag KC Stuttgart · New York ISSN 1434-9132 


\section{Frage 1}

Welches der folgenden Insuline hat bei der Katze intermediäre Wirkdauer?
A Glargin
B Detemir
C Protamin-Zink-Insulin
D porcines Lente-Insulin
E Normalinsulin

\section{Frage 2}

Welches der folgenden Insuline ist am potentesten?
A Glargin
B Detemir
C Protamin-Zink-Insulin
D porcines Lente-Insulin
E Normalinsulin

\section{Frage 3}

Welches der folgenden Insuline wird an Albumin gebunden, um eine längere Wirkdauer zu gewährleisten?
A Glargin
B Detemir
C Protamin-Zink-Insulin
D porcines Lente-Insulin
E Normalinsulin

\section{Frage 4}

In welchem Abstand sollte man im Idealfall, bei einem unkomplizierten Diabetiker, die Insulindosis in der Stabilisierungsphase anpassen?
A jeden Tag
$B$ alle 3 Tage
$C$ alle 5-7 Tage
D alle 3 Wochen
E alle 4-6 Wochen

\section{Frage 5}

Welche der folgenden Monitoring-Methoden ist am wenigsten geeignet, um eine Insulindosis anzupassen?

A Blutzuckertagesprofil, angefertigt zu Hause

B Blutzuckertagesprofil, angefertigt in der Praxis

C Fruktosamine

D kontinuierliches Glukosemonitoring

E Einzelmessung der Glukose zum geschätzten Nadir-Zeitpunkt

\section{Frage 6}

Welche Blutglukose-Konzentration entspricht ungefähr der Nierenschwelle bei der Katze?
A $8 \mathrm{mmol} / \mathrm{l}$
B $12 \mathrm{mmol} / \mathrm{l}$
C $15 \mathrm{mmol} / \mathrm{l}$
D $20 \mathrm{mmol} / \mathrm{l}$
E $25 \mathrm{mmol} / \mathrm{l}$

\section{Frage 7}

Welcher Glukose-Nadir wird empfohlen bei der Katze?
A $3-5 \mathrm{mmol} / \mathrm{l}$
B $4,5-8 \mathrm{mmol} / \mathrm{l}$
C $8-10,5 \mathrm{mmol} / \mathrm{l}$
D $12-15 \mathrm{mmol} / \mathrm{l}$
E $15-17 \mathrm{mmol} / \mathrm{l}$

\section{Frage 8}

Die Insulindosis sollte schrittweise angepasst werden. Besteht der Bedarf für eine Insulindosis-Erhöhung, in welchen Schritten sollte diese idealerweise erfolgen?
A $0,5 \mathrm{IE} / \mathrm{kg} \mathrm{q} 12 \mathrm{~h}$
B $0,5 \mathrm{IE} /$ Injektion q $24 \mathrm{~h}$
C $0,5 \mathrm{IE} /$ Injektion q $12 \mathrm{~h}$
D 2 IE/Injektion q $12 \mathrm{~h}$
E $1,5 \mathrm{IE} / \mathrm{kg} \mathrm{q} 12 \mathrm{~h}$

\section{Frage 9}

Eine Hypoglykämie stellt die häufigste Nebenwirkung der Insulintherapie dar. Der sog. Somogyi-Effekt kommt jedoch sehr selten vor und in einer kürzlich publizierten Studie wurde vorgeschlagen, die Bezeichnung durch „posthypoglykämische Hyperglykämie“" zu ersetzen. Welches der folgenden Resultate hat diese Studie gezeigt? Eine posthypoglykämische Hyperglykämie wurde assoziiert mit:
A erhöhten Remissionsraten
B höherem Alter
C männlichem Geschlecht
D höherem Körpergewicht
E schlechteren glykämischen Kontrolle

\section{Frage 10}

Durch welche der folgenden Behandlungsmöglichkeiten kann die Insulintherapie bei der Katze ersetzt werden?
A Metformin
B Glipizide
C kohlenhydratreiche Diät
D Exenatid
E kein Ersatz möglich 
Insulintherapie bei der Katze Tipps für die richtige Insulindosis

Katarina Hazuchova

\section{A Lernerfolgskontrolle}

\section{Bitte kreuzen Sie die richtigen \\ Antworten an! Es ist jeweils nur 1 Antwort pro Frage richtig!}

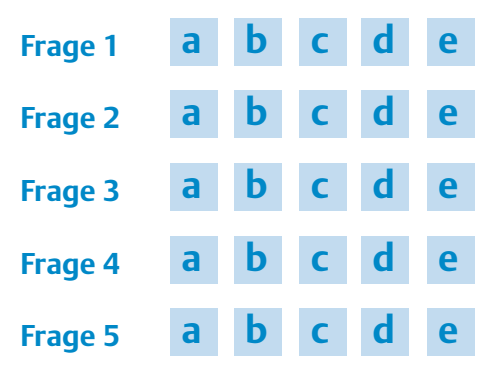

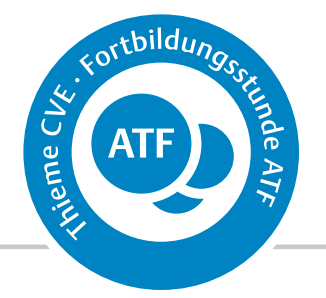

\section{B Teilnehmer}

Titel | Name | Vorname

Straße | Hausnummer

PLZ | Ort

Beruf

\section{Ihr Ergebnis wird vom Verlag ausgefültt}

Sie haben von Fragen

$\square$ bestanden und 1 ATF-Stunde erhalten

Stuttgart, den

\begin{tabular}{l|l|l|l|l|l|l|} 
Frage 6 & a & b & c & d & e \\
\hline Frage 7 & a & b & c & d & e \\
\hline Frage 8 & a & b & c & d & e \\
\hline Frage 9 & a & b & c & d & e \\
\hline Frage 10 & a & b & c & d & e \\
\hline
\end{tabular}

richtig beantwortet und somit

\section{nnenten der kleintier.konkret kostenlos}

\section{Teilnahmebedingungen}

Für diese Fortbildung können Sie 1 ATF-Fortbildungsstunde anerkannt bekommen. Hierfür ...

- müssen mindestens $70 \%$ der Fragen richtig beantwortet sein.

- muss der Antwortbogen vollständig ausgefüllt sein. Unvollständig ausgefüllte Bögen können nicht berücksichtigt werden.

\begin{abstract}
- muss im markierten Feld* Ihre Abonnentennummer eingetragen oder eine Kleintier konkret-CVE-Wertmarke aufgeklebt sein.
\end{abstract}

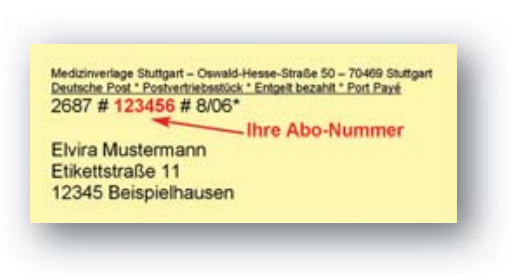

Kleintier konkret-CVE-Wertmarken für Nichtabonnenten können beim Verlag zu folgenden Bedingungen erworben werden: 6er-Pack Wertmarken, Preis 49,95€ inkl. MwSt., ArtikelNr. 903000. Bitte richten Sie die Bestellungen an: Georg Thieme Verlag KG, KundenServiceCenter Buch, Postfach 301120, 70451 Stuttgart.

* Nichtabonnenten bitte hier Kleintier-konkret-CVE-Wertmarke aufkleben. Abonnenten bitte Abonnentennummer eintragen: 